\title{
Topological crystalline superconductivity in locally noncentrosymmetric $\operatorname{CeRh}_{2} \mathrm{As}_{2}$
}

\author{
Kosuke Nogaki $\odot,{ }^{1, *}$ Akito Daido $\odot,{ }^{1}$ Jun Ishizuka $\odot,{ }^{1}$ and Youichi Yanase ${ }^{1,2}$ \\ ${ }^{1}$ Department of Physics, Kyoto University, Kyoto 606-8502, Japan \\ ${ }^{2}$ Institute for Molecular Science, Okazaki 444-8585, Japan
}

(Received 8 March 2021; revised 4 August 2021; accepted 6 August 2021; published 23 September 2021)

\begin{abstract}
Recent discovery of superconductivity in $\mathrm{CeRh}_{2} \mathrm{As}_{2}$ clarified an unusual $H$ - $T$ phase diagram with two superconducting phases [Khim et al. Science, 373, 1012 (2021)]. The experimental observation has been interpreted based on the even-odd parity transition characteristic of locally noncentrosymmetric superconductors. Indeed, inversion symmetry is locally broken at the Ce site, and $\mathrm{CeRh}_{2} \mathrm{As}_{2}$ molds a class of exotic superconductors. The low-temperature and high-field superconducting phase is a candidate for the odd-parity pair-density-wave state, suggesting a possibility of topological superconductivity like spin-triplet superconductors. In this Letter, we first derive the formula expressing the $\mathbb{Z}_{2}$ invariant of glide symmetric and time-reversal symmetry-broken superconductors by the number of Fermi surfaces on a glide invariant line. Next, we conduct first-principles calculations for the electronic structure of $\mathrm{CeRh}_{2} \mathrm{As}_{2}$. Combining the results, we show that the field-induced oddparity superconducting phase of $\mathrm{CeRh}_{2} \mathrm{As}_{2}$ is a platform of topological crystalline superconductivity protected by nonsymmorphic glide symmetry and accompanied by boundary Majorana fermions.
\end{abstract}

DOI: 10.1103/PhysRevResearch.3.L032071

\section{INTRODUCTION}

For decades, symmetry breaking has been the most important concept to describe various phases of quantum matter such as magnetism, density waves, and superconductivity as well as critical phenomena in condensed matter physics [1]. Recently, topological science has shed light on other aspects [2-5]. Topological phase transitions without symmetry breaking have been uncovered, and topological insulators/superconductors are widely recognized as intriguing phases of matter. One of the exotic phenomena in topological materials is the appearance of gapless modes at boundaries and defects of the systems, though the topology is determined by only bulk information. Topological superconductors host Majorana fermions, which have been proposed for topological fault-tolerant quantum computation [6,7]. Such unique properties, attractive from the viewpoints of basic and applied science, triggered tremendous efforts for searching for topological superconductivity [8-45]. However, the realization of topological superconductivity is still under intensive debate.

By pioneering research [46-48], the topological phases were classified based on local symmetries, namely, timereversal, particle-hole, and chiral symmetries. It has later been recognized that symmetries unique to solids enrich the topological properties of materials. This idea led to the

\footnotetext{
*nogaki.kosuke.83v@st.kyoto-u.ac.jp

Published by the American Physical Society under the terms of the Creative Commons Attribution 4.0 International license. Further distribution of this work must maintain attribution to the author(s) and the published article's title, journal citation, and DOI.
}

concept of topological crystalline insulators/superconductors (TCIs/TCSCs) [49-70]. However, major candidates of TCSCs are odd-parity superconductors, as is the case for usual topological superconductors [3-5]. Unfortunately, the odd-parity superconductors are usually spin-triplet superconductors, which are rarely known in nature. On the other hand, superconductivity with exotic symmetry beyond the paradigm of standard classification theory [71] has recently attracted attention, and odd-parity superconductivity due to an ordinary spin-singlet pairing has been reported in $\mathrm{CeRh}_{2} \mathrm{As}_{2}$ [72]. This discovery may open a route to realize topological superconductivity.

This Letter was triggered by a recent experimental report of an unusual superconducting $H-T$ phase diagram in a heavyfermion superconductor $\mathrm{CeRh}_{2} \mathrm{As}_{2}$ [72]. A high upper critical field much beyond the Pauli-Clogston-Chandrasekhar limit and the phase transition between two superconducting phases have been observed [Fig. 1(a)]. Thus, $\mathrm{CeRh}_{2} \mathrm{As}_{2}$ is a fascinating platform of multiple superconducting phases. Different from the previous examples, $\mathrm{UPt}_{3}$ [73] and $\mathrm{UTe}_{2}$ [74-77], the phase diagram was attributed to the locally noncentrosymmetric crystal structure [72] in accordance with a theoretical proposal [78]. Soon after the experimental report, theoretical works along this line were conducted [79-82].

Noncentrosymmetric superconductivity in global inversion asymmetric systems have been investigated for several decades [9-13,43,44,83-92]. The concept was recently extended to locally noncentrosymmetric superconductivity, and unique superconducting phenomena have been uncovered [57,78,93-101]. The $H-T$ phase diagram of $\mathrm{CeRh}_{2} \mathrm{As}_{2}$ is consistent with the prediction based on a two-sublattice Rashba model $[78,100]$. The similarity of phase diagrams between experiment [72] and theory [78,100] suggests that local inversion symmetry breaking plays an essential role in 

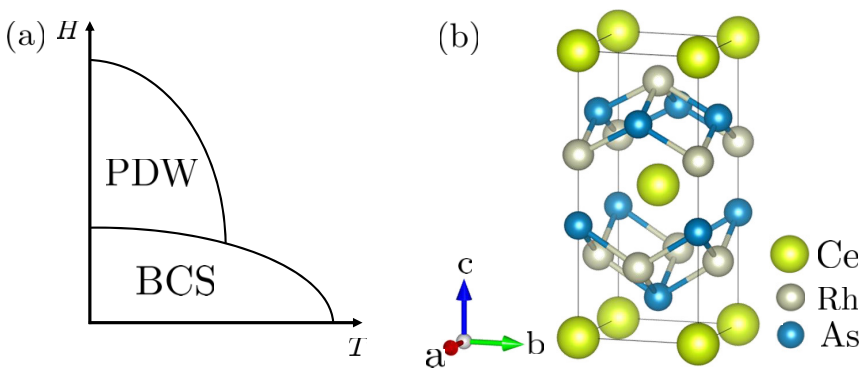

FIG. 1. (a) Schematic $H-T$ phase diagram of $\mathrm{CeRh}_{2} \mathrm{As}_{2}$ [72]. The Bardeen-Cooper-Schrieffer state is assumed to be an even-parity superconducting phase, and the high-field phase is supposed to be the pair-density-wave (PDW) state, the main topic in this Letter. (b) Crystal structure of $\mathrm{CeRh}_{2} \mathrm{As}_{2}$ generated by VESTA [104].

$\mathrm{CeRh}_{2} \mathrm{As}_{2}$, and the superconducting phase in the high magnetic field region is the pair-density-wave (PDW) state. In the PDW state, the superconducting gap function changes sign depending on Ce layers [102]. The most crucial property of this phase is the odd-parity superconductivity despite dominant spin-singlet pairing. The odd parity is owing to the sign change of the gap function between the sublattices related by inversion symmetry [78]. Thus, the locally noncentrosymmetric crystal is a platform of odd-parity superconductivity without requiring rare spin-triplet pairing. It may be realized in $\mathrm{CeRh}_{2} \mathrm{As}_{2}$, making a candidate of the topological superconductor different from potential spin-triplet superconductors $\mathrm{UPt}_{3}[56,62], \mathrm{UCoGe}[63]$, and $\mathrm{UTe}_{2}$ [103].

\section{CRYSTAL STRUCTURE AND GLIDE SYMMETRY}

$\mathrm{CeRh}_{2} \mathrm{As}_{2}$ crystallizes in the centrosymmetric tetragonal $\mathrm{CaBe}_{2} \mathrm{Ge}_{2}$-type structure [72] with stacking Ce layers and $\mathrm{Rh}_{2} \mathrm{As}_{2}$ layers [Fig. 1(b)]. Importantly, $\mathrm{Rh}_{2} \mathrm{As}_{2}$ layers at the top and bottom of the Ce layer have different compositions. Therefore, inversion symmetry is locally broken at the $\mathrm{Ce}$ sites, although the global inversion center exists in the middle of the two Ce sites in the unit cell. The space group is $\mathrm{P} 4 / \mathrm{nmm}$ (No. 129) including one glide reflection and three screw rotations. Since the magnetic field parallel to the $c$ axis destroys the three screw symmetries, only glide symmetry is respected in the field-induced phase, and we hereafter focus on the glide operation $\hat{G}$, which is $\left\{M_{z} \mid \mathbf{a} / 2+\mathbf{b} / 2\right\}$ in Seitz notation. Here, $\mathbf{a}$ and $\mathbf{b}$ are the lattice vectors along the $a$ and $b$ axes, respectively.

In the Brillouin zone, glide symmetry is preserved in the two glide-invariant planes $k_{z}=0, \pi$, and we can divide the Hilbert space into each Bloch state and glide sector:

$$
\mathbb{V}_{k_{z}=0, \pi}=\bigoplus_{\mathbf{k} \in \mathrm{BZ}_{k_{z}=0, \pi}} \mathbb{V}_{\mathbf{k}}^{\mathfrak{g}^{+}} \oplus \mathbb{V}_{\mathbf{k}}^{\mathfrak{g}^{-}} .
$$

Here, $\mathbb{V}_{\mathbf{k}}$ is the Hilbert space consisting of Bloch states labeled by $\mathbf{k}$, and $\mathfrak{g}^{ \pm}$are eigenvalues of the glide operation. Because the Hamiltonian preserves glide symmetry $[\hat{H}, \hat{G}]=0$, we can label eigenstates of the Hamiltonian by glide eigenvalues as $\left|\mathbf{k}^{\mathfrak{g}^{ \pm}}\right\rangle \in \mathbb{V}_{\mathbf{k}}^{\mathfrak{g}^{ \pm}}$. To evaluate the eigenvalues in the spinful case, we focus on the following relation:

$$
\hat{G}^{2}=\{-E \mid \mathbf{a}+\mathbf{b}\} \text {. }
$$

Owing to Eq. (2), any state $|\mathbf{k}\rangle$ in $\mathbb{V}_{k_{z}=0, \pi}$ satisfies

$$
\hat{G}^{2}|\mathbf{k}\rangle=-\exp \left[-i\left(k_{x}+k_{y}\right)\right]|\mathbf{k}\rangle,
$$

and we conclude that the glide eigenvalues are

$$
\mathfrak{g}^{ \pm}= \pm i \exp \left[-\frac{i\left(k_{x}+k_{y}\right)}{2}\right] \text {. }
$$

\section{SYMMETRY OF SUPERCONDUCTIVITY}

Superconductivity is classified by the point group $D_{4 h}$, which has 8 one-dimensional (1D) irreducible representations and 2 two-dimensional (2D) ones. Many Ce-based heavy-fermion systems undergo spin-singlet superconductivity, which is also expected in $\mathrm{CeRh}_{2} \mathrm{As}_{2}$ at $H=0$. Thus, the low-field superconducting phase is supposed to be evenparity, either of $A_{1 g}, A_{2 g}, B_{1 g}, B_{2 g}$, or $E_{g}$ state. Accordingly, the high-field phase is an odd-parity $A_{1 u}, A_{2 u}, B_{1 u}, B_{2 u}$, or $E_{u}$ state when the PDW state is assumed. Hereafter, we focus on $1 \mathrm{D}$ odd-parity representations because the $E_{u}$ state is unlikely in $\mathrm{CeRh}_{2} \mathrm{As}_{2}$ [72].

\section{IV. $\mathbb{Z}_{2}$ INVARIANTS}

Let us discuss the $\mathbb{Z}_{2}$ topological invariants protected by glide symmetry. By definition, the PDW state is glide-odd superconductivity in which the superconducting gap function obeys the following relation:

$$
\mathcal{G}(\mathbf{k}) \Delta(\mathbf{k}) \mathcal{G}^{\top}(-\mathbf{k})=-\Delta\left(M_{z} \mathbf{k}\right) .
$$

Here, $\mathcal{G}(\mathbf{k})$ is the representation matrix of the glide operation $\hat{G}$ in the Hilbert space $\mathbb{V}_{\mathbf{k}}, M_{z} \mathbf{k} \equiv\left(k_{x}, k_{y},-k_{z}\right)$, and $\Delta(\mathbf{k})$ represents the gap function.

In the following, we focus on the glide invariant planes in the Brillouin zone $k_{z}=0, \pi$. From Eq. (5), the particle-hole operation $\hat{C}$ and glide operation $\hat{G}$ anticommutes $\{\hat{C}, \hat{G}\}=$ 0 , and from Eq. (4), the glide eigenvalues become pure imaginary $\pm i$ in the restricted Hilbert space on $k_{x}+k_{y}=0$. Combining these results, we show that the particle-hole symmetry is closed in each glide sector:

$$
\hat{G} \hat{C}\left|\mathbf{k}^{\mathfrak{g}^{ \pm}}\right\rangle=-\hat{C}( \pm i)\left|\mathbf{k}^{\mathfrak{g}^{ \pm}}\right\rangle=( \pm i) \hat{C}\left|\mathbf{k}^{\mathfrak{g}^{ \pm}}\right\rangle .
$$

As the time-reversal symmetry is broken under the magnetic field, each glide sector on the line is classified into 1D class D superconductivity. This class is specified by the topological invariant $\mathbb{Z}_{2}$, which is given by the integral of the Berry connection as introduced in Ref. [60]:

$$
v^{\mathfrak{g}^{ \pm}}=\frac{1}{\pi} \int_{\Gamma_{1}}^{\Gamma_{2}} d k_{i} \mathcal{A}_{i}^{\mathfrak{g}^{ \pm}}(\mathbf{k}) \quad(\bmod 2),
$$

where $\mathcal{A}_{i}^{\mathfrak{g}^{ \pm}}(\mathbf{k})$ are $i$ th components of the Berry connection of each glide sector given by $i \sum_{\text {occ. }}\left\langle\psi_{\mathbf{k}}^{\mathfrak{g}^{ \pm}}\left|\partial_{k_{i}}\right| \psi_{\mathbf{k}}^{\mathfrak{g}^{ \pm}}\right\rangle$. Here, $\left|\psi_{\mathbf{k}}^{\mathfrak{g}^{ \pm}}\right\rangle$are the occupied eigenstates of the Bogoliubov-de Gennes $(\mathrm{BdG})$ Hamiltonian in each glide sector with the glide eigenvalue $\mathfrak{g}^{ \pm}$. The time-reversal invariant momenta (TRIM) are $\Gamma_{1}=(0,0,0)(\Gamma$ point $)$ or $(0,0, \pi)(Z$ point $)$ and $\Gamma_{2}=$ $(\pi,-\pi, 0)(M$ point $)$ or $(\pi,-\pi, \pi)(A$ point $)$, depending on $k_{z}=0$ or $\pi$.

In addition to the glide $\mathbb{Z}_{2}$ invariants $v^{\mathfrak{g}^{ \pm}}$, we can define the Chern number $C$ as well as two Zak phases $\gamma$ and $\gamma^{\prime}$ on the 
lines $k_{x}+k_{y}=\pi$ and 0 , respectively. However, they are not independent of each other. Actually, $\gamma^{\prime}=v^{\mathfrak{g}^{+}}+v^{\mathfrak{g}^{-}}$holds, and later, we will show $\gamma=0$. Thus, the topology of the PDW state for $k_{z}=0, \pi$ can be characterized by identifying $\nu^{\mathfrak{g}^{ \pm}}$and $C$ (denoted by $v_{0, \pi}^{\mathfrak{g}^{ \pm}}$and $C_{0, \pi}$ for each $k_{z}$ ). Furthermore, the Chern number satisfies the relation

$$
\begin{aligned}
C & =\gamma^{\prime}-\gamma \quad(\bmod 2), \\
& =v^{\mathfrak{g}^{+}}+v^{\mathfrak{g}^{-}} \quad(\bmod 2) .
\end{aligned}
$$

Therefore, only one of $\nu^{\mathfrak{g}^{ \pm}}$is a strong topological index in accordance with $K$-theory classification for the strong indices $\mathbb{Z} \oplus \mathbb{Z}_{2}$ [60]. In this Letter, we discuss $\nu^{\mathfrak{g}^{ \pm}}$and $C$ modulo two in $\mathrm{CeRh}_{2} \mathrm{As}_{2}$ since they do not rely on the details of the order parameter, as we see below. They are determined only by the topology of the Fermi surfaces and do not require the full calculation of the symmetry indicators [64-70].

Next, to simplify the expression in Eq. (7), we pay attention to the following relation:

$$
\hat{I} \hat{G}=\hat{G} \hat{I}\{E \mid \mathbf{a}+\mathbf{b}\},
$$

where $\hat{I}$ is the space inversion operation. As $\{E \mid \mathbf{a}+\mathbf{b}\}$ is reduced to merely a phase factor $\exp \left[-i\left(k_{x}+k_{y}\right)\right], \hat{I}$ and $\hat{G}$ commute in the Hilbert space $\mathbb{V}_{k_{x}+k_{y}=0, k_{z}=0, \pi}$. Therefore, the space inversion parity is well defined in the glide sector, and each glide sector corresponds to a 1D class D odd-parity superconductor. We can use the Fermi surface formula for odd-parity superconductors $[31,32]$, by which the $\mathbb{Z}_{2}$ invariant is evaluated based on the topology of Fermi surfaces. As a consequence, we finally get the conclusion that $\nu^{\mathfrak{g}^{ \pm}}$is nontrivial (trivial) when the number of Fermi surfaces between $\Gamma_{1}$ and $\Gamma_{2}$ is odd (even):

$$
\begin{array}{ll}
v_{0}^{\mathfrak{g}^{ \pm}}=\# \mathrm{FS}_{\Gamma \rightarrow M}^{ \pm} & (\bmod 2), \\
v_{\pi}^{\mathfrak{g}^{ \pm}}=\# \mathrm{FS}_{Z \rightarrow A}^{ \pm} & (\bmod 2) .
\end{array}
$$

In a similar way, we can relate $\gamma$ to the number of Fermi surfaces. Because the BdG Hamiltonian on the line $k_{x}+k_{y}=$ $\pi$ can be regarded as a 1D odd-parity superconductor, the Fermi surface formula [31,32] reads

$$
\gamma_{0, \pi}=\# \mathrm{FS}_{\Gamma_{1} \rightarrow \Gamma_{2}} \quad(\bmod 2) .
$$

Here, $\Gamma_{1}=X, R$ and $\Gamma_{2}=X^{\prime}, R^{\prime}$ for $k_{z}=0, \pi$, respectively. The fourfold rotation symmetry $C_{4}^{z}$ forces the occupation numbers of electrons at $\Gamma_{1}$ and $\Gamma_{2}$ to be equal. Therefore, the electron bands must cross the Fermi level even times between $\Gamma_{1}$ and $\Gamma_{2}$, leading to $\gamma_{0, \pi}=0$.

Below, we conduct first-principles calculations for $\mathrm{CeRh}_{2} \mathrm{As}_{2}$ and evaluate the $\mathbb{Z}_{2}$ invariants. The first-principles calculations are carried out at the zero magnetic field, where all the electronic bands are doubly degenerate $\left|\mathbf{k}^{\mathfrak{g}^{ \pm}}\right\rangle$ and $\hat{I} \hat{\Theta}\left|\mathbf{k}^{\mathfrak{g}^{ \pm}}\right\rangle$because of the inversion symmetry $\hat{I}$ and time-reversal symmetry $\hat{\Theta}$. The relation

$$
\hat{G} \hat{I} \hat{\Theta}\left|\mathbf{k}^{\mathfrak{g}^{ \pm}}\right\rangle=\hat{I} \hat{\Theta} \hat{G}\left|\mathbf{k}^{\mathfrak{g}^{ \pm}}\right\rangle=\mathfrak{g}^{\mp} \hat{I} \hat{\Theta}\left|\mathbf{k}^{\mathfrak{g}^{ \pm}}\right\rangle,
$$

reveals that the degenerate states belong to different glide sectors. Consequently, the numbers of Fermi surfaces are equivalent between the two glide sectors and coincide with the number of spinful bands crossing the Fermi level $\# \mathrm{FS}_{\Gamma_{1} \rightarrow \Gamma_{2}}^{+}=$
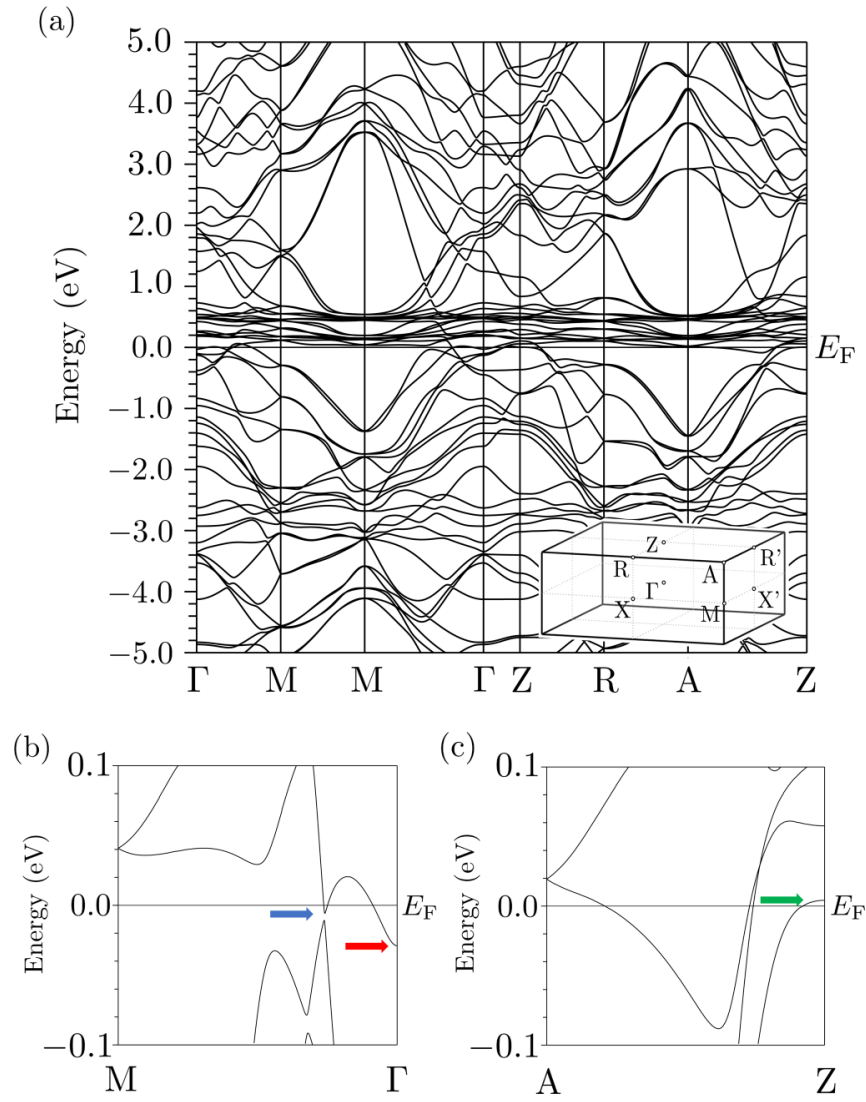

FIG. 2. Electronic band structure of $\mathrm{CeRh}_{2} \mathrm{As}_{2}$ with spin-orbit coupling calculated by WIEN2k. (a) The whole bands along symmetric lines. The heavy bands of Ce $4 f$ electrons are seen near the Fermi level. (b) Enlarged view along the $M-\Gamma$ line. The bands cross the Fermi level three times. (c) Enlarged view along the $A-Z$ line. The bands cross the Fermi level four times.

$\# \mathrm{FS}_{\Gamma_{1} \rightarrow \Gamma_{2}}^{-} \equiv \mathrm{FFS}_{\Gamma_{1} \rightarrow \Gamma_{2}}$. The magnetic field does not alter the $\mathbb{Z}_{2}$ invariants and Chern number modulo two unless it causes the Lifshitz transition. We will discuss the effect of possible Lifshitz transitions later.

\section{BAND STRUCTURE OF CeRh $\mathrm{As}_{2}$}

We carry out density functional theory (DFT) for electronic structure calculations using the WIEN2k package [106]. The crystallographic parameters are experimentally obtained values [72]. We employ the full-potential linearized augmented plane wave + local orbitals method within the generalized gradient approximation with the spin-orbit coupling. Details of the band calculations, density of states, and orbital weights for each atom are given in the Supplemental Material [107]. Figure 2(a) shows the band structure of $\mathrm{CeRh}_{2} \mathrm{As}_{2}$, revealing the heavy bands of $\mathrm{Ce} 4 f$ electrons near the Fermi level. The main contribution to the density of states at the Fermi level comes from the Ce $4 f$ orbitals [107]. We show the Fermi surfaces in Fig. 3. The Ce $4 f$ electrons with hybridization to $\mathrm{Rh} 4 d_{x^{2}-y^{2}}$ electrons mainly constitute the Fermi surface except for the pink pockets near the $A$ point [Fig. 3(d)]. The largest Fermi surface [Fig. 3(c)] is quasi-2D, supporting the field-induced PDW state [78]. In the experiments, the Kondo 


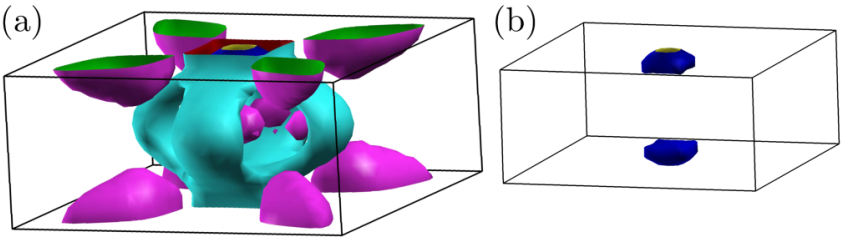

(c)

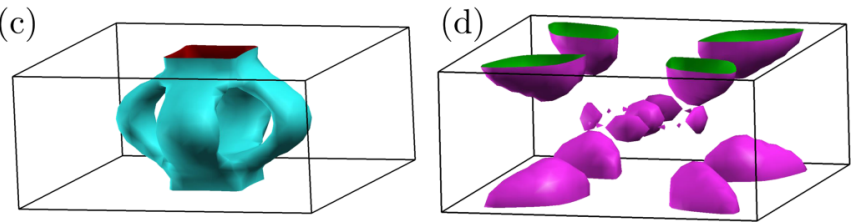

FIG. 3. (a) Fermi surfaces of $\mathrm{CeRh}_{2} \mathrm{As}_{2}$. Each Fermi surface is shown in (b)-(d). The Fermi surfaces other than the pockets around the $A$ point in (d) are mainly constituted by Ce $4 f$ electrons. The figures are generated by XCRYSDEN software [105].

effect was observed [72,108], and the electronic specific heat coefficient $\gamma$ was $\sim 1000 \mathrm{~mJ} / \mathrm{mol} \mathrm{K}^{2}$, which support the presence of heavy-fermion bands in $\mathrm{CeRh}_{2} \mathrm{As}_{2}$. Therefore, our result seems to be consistent with experiments.

Hereafter, we proceed with discussions based on DFT calculations. The formulas in Eqs. (11) and (12) of $\mathbb{Z}_{2}$ invariants are universal, and we can judge whether $\mathrm{CeRh}_{2} \mathrm{As}_{2}$ is TCSC when the topology of Fermi surfaces is determined. Although we keep in mind the spin-singlet pairing dominant PDW state, essential properties for the Fermi surface formula are the odd-parity and glide-odd nature of the PDW state. These properties are ensured by the negative character of the inversion operation and the glide operation for $A_{1 u}, A_{2 u}, B_{1 u}$, and $B_{2 u}$ states regardless of whether the spin-singlet component or the spin-triplet component is dominant. Therefore, our formula also applies to spin-triplet dominant states, such as proposed in Ref. [79]. Since the magnitude of the spin-triplet channel affects the physical properties such as the paramagnetic effect and the local density of states [97], and these quantities can be measured in principle, further theoretical and experimental efforts are desired to clarify the detail of the high-field phase. However, the following results are not affected by the details.

\section{TCSC IN CeRh $\mathrm{Ass}_{2}$}

We now show the $\mathbb{Z}_{2}$ invariants. First, we discuss the topology on a glide-invariant plane $k_{z}=0$. Focusing on the $\Gamma-M$ line [Fig. 2(b)], we notice that bands cross the Fermi level three times. Therefore, both $v_{0}^{\mathfrak{g}^{ \pm}}$are nontrivial, revealing the TCSC. The TCSC is robust against the Zeeman splitting. In $\mathrm{CeRh}_{2} \mathrm{As}_{2}$, the PDW state is observed under the magnetic field between 4 and $14 \mathrm{~T}$ [72], in which the Zeeman splitting energy is estimated as $2-8 \mathrm{meV}$ when we assume a probably overestimated $g$ factor $g=10$. At the $\Gamma$ point (red arrow), the conduction band is $\sim 30 \mathrm{meV}$ below the Fermi level, and thus, the occupation number does not change. Although the crossing points indicated by the blue arrow may be lifted, it does not influence the $\mathbb{Z}_{2}$ invariants according to the formula in Eq. (11). Therefore, the number of Fermi surfaces modulo two remains the same under the magnetic field, and the $\mathbb{Z}_{2}$ invariants $v_{0}^{\mathfrak{g}^{ \pm}}$are nontrivial in the PDW state.
TABLE I. $\mathbb{Z}_{2}$ topological invariants and Chern number of $\mathrm{CeRh}_{2} \mathrm{As}_{2}$ in the PDW state based on first-principles calculations. A possibility of a Weyl superconducting state is shown. In the high-field region, one of the Zeeman split bands is supposed to cause a Lifshitz transition at the $Z$ point.

\begin{tabular}{lccc}
\hline \hline & $\left(v_{0}^{\mathfrak{g}^{+}}, v_{0}^{\mathfrak{g}^{-}}, C_{0}\right)$ & $\left(v_{\pi}^{\mathfrak{g}^{+}}, v_{\pi}^{\mathfrak{g}^{-}}, C_{\pi}\right)$ & Weyl SC \\
\hline Low field & $(1,1$, even $)$ & $(0,0$, even $)$ & $\times$ \\
High field & $(1,1$, even $)$ & $(1,0$, odd $)$ or $(0,1$, odd $)$ & $\circ$ \\
\hline \hline
\end{tabular}

Next, we discuss the other glide-invariant plane $k_{z}=\pi$. On the $Z$ - $A$ line [Fig. 2(c)], bands cross the Fermi level four times, and accordingly, $\nu_{\pi}^{\mathfrak{g}^{ \pm}}$are trivial. However, a hole band (green arrow) is shallow, and the energy at the $Z$ point is estimated to be $\sim 4 \mathrm{meV}$. Therefore, the Lifshitz transition may occur under the magnetic field, and the $\mathbb{Z}_{2}$ invariants may become nontrivial. The $\mathbb{Z}_{2}$ topological invariants of $\mathrm{CeRh}_{2} \mathrm{As}_{2}$ are summarized in Table I based on the band structure calculation. We assume that one of the Zeeman split bands undergoes the Lifshitz transition at the $Z$ point in the high-field region. Then the Chern number as well as a $\mathbb{Z}_{2}$ invariant is nontrivial. Because the Chern number is well-defined on any constant $k_{z}$ plane, the difference in $C_{0}$ and $C_{\pi}$ indicates a Weyl superconducting state as in $\mathrm{UPt}_{3}$ [109].

From these results, $\mathrm{CeRh}_{2} \mathrm{As}_{2}$ is shown to be a platform of TCSC. We would like to stress that the glide $\mathbb{Z}_{2}$ invariants are nontrivial at least on the $k_{z}=0$ plane. Therefore, boundary Majorana states appear at the $(\overline{1} 10)$ surface preserving glide symmetry, which is generated by $\mathbf{a}+\mathbf{b}$ and $\mathbf{c}$.

\section{MODEL STUDY}

To demonstrate the emergence of Majorana surface states, we conducted numerical calculations based on a tight-binding model. We construct the tight-binding model and set parameters [110] so that $\# \mathrm{FS}_{\Gamma_{1} \rightarrow \Gamma_{2}}^{ \pm}$are odd (even) at $k_{z}=0\left(k_{z}=\pi\right)$, consistent with first-principles calculations. In the spectrum for $k_{z}=0$ (Fig. 4), we recognize the stable Majorana surface states for both $A_{2 u}$ representation ( $s+p$-wave state) and $B_{2 u}$ representation $\left(d_{x^{2}-y^{2}}+p\right.$-wave state). We also verified the Majorana states for the $A_{1 u}$ and $B_{1 u}$ representations, while the surface states are gapped on the other glide-invariant plane
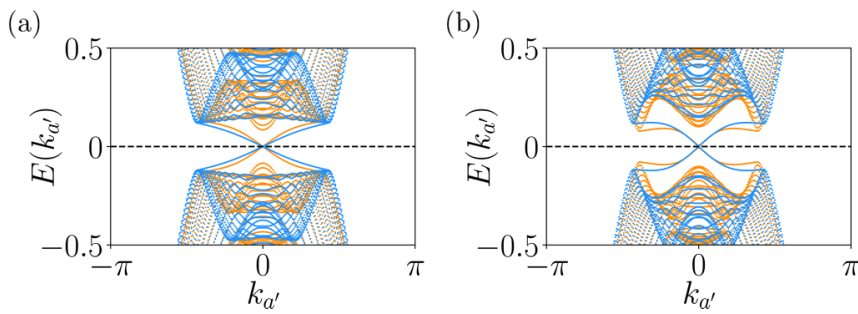

FIG. 4. (110) and (11̄0) surface states of (a) $A_{2 u}$ and (b) $B_{2 u}$ superconducting states at one of the glide-invariant planes, $k_{z}=0$. The orange (blue) color represents the glide-even (odd) sector. The axis $k_{a^{\prime}}$ corresponds to $k_{x}+k_{y}$, and Majorana surface states appear at $k_{a^{\prime}}=0$. 
$k_{z}=\pi$ (see the Supplemental Material [110]). All results are consistent with the topological invariants discussed above.

\section{SUMMARY AND CONCLUSIONS}

In this Letter, we theoretically investigated the electronic structure and topological superconductivity in a recently discovered heavy-fermion superconductor $\mathrm{CeRh}_{2} \mathrm{As}_{2}$. First, using group theory, we clarified the algebra of symmetry operations in the Bloch representation and decomposed the Hilbert space on the glide-invariant planes $k_{z}=0, \pi$ into the glide sectors. Supposing the PDW state in the high-field superconducting phase as proposed, based on these results, we derived the Fermi surface formula of $\mathbb{Z}_{2}$ invariants specifying the TCSC protected by nonsymmorphic glide symmetry. Second, we conducted first-principles calculations for the electronic structure of $\mathrm{CeRh}_{2} \mathrm{As}_{2}$. Evaluating the $\mathbb{Z}_{2}$ invariants, we found the TCSC due to heavy-fermion bands of $\mathrm{Ce} 4 f$ electrons hybridizing with conduction electrons. The emergence of Majorana fermions at the surface preserving glide symmetry was demonstrated based on the tight-binding model. This Letter proposes $\mathrm{CeRh}_{2} \mathrm{As}_{2}$ as a class of topological superconductors in the following two senses: (1) The topological superconductivity requires neither spin-triplet pairing nor topological band structures. (2) The topological structure is protected by nonsymmorphic symmetry, which does not have a counterpart in continuous systems.

Note added. Recently, two different groups independently reported the results of the electronic structure calculation of $\mathrm{CeRh}_{2} \mathrm{As}_{2}$ using the pseudopotential and full potential method [81,82]. Their results are consistent with our results and support our conclusion. In Ref. [81], the authors discuss the Lifshitz transition and orbital order using VASP. In Ref. [82], combining $\mathbf{k} \cdot \mathbf{p}$ theory and DFT calculation, the dominance of the Rashba type spin-orbit coupling around the Dirac line and the origin of the large critical field are discussed.

\section{ACKNOWLEDGMENTS}

The authors are grateful to S. Sumita, H. Watanabe, T. Kitamura, S. Khim, C. Geibel, and K. Ishida for fruitful discussions. We thank S. Khim and C. Geibel for providing the parameters of the crystal structure before the submission of their paper. Some figures in this Letter were created by using VESTA [104] and XCRYSDEN [105]. This Letter was supported by JSPS KAKENHI (Grants No. JP18H05227, No. JP18H01178, and No. 20H05159), SPIRITS 2020 of Kyoto University, and Research Grants, 2020 of WISE Program, MEXT
[1] L.D. Landau and E.M. Lifshitz, Statistical Physics: Volume 5 (Elsevier Science, London, 2013).

[2] X.-L. Qi and S.-C. Zhang, Topological insulators and superconductors, Rev. Mod. Phys. 83, 1057 (2011).

[3] Y. Tanaka, M. Sato, and N. Nagaosa, Symmetry and topology in superconductors - odd-frequency pairing and edge states, J. Phys. Soc. Jpn. 81, 011013 (2012).

[4] M. Sato and S. Fujimoto, Majorana fermions and topology in superconductors, J. Phys. Soc. Jpn. 85, 072001 (2016).

[5] M. Sato and Y. Ando, Topological superconductors: A review, Rep. Prog. Phys. 80, 076501 (2017).

[6] A. Yu. Kitaev, Unpaired Majorana fermions in quantum wires, Phys. Usp. 44, 131 (2001).

[7] C. Nayak, S. H. Simon, A. Stern, M. Freedman, and S. Das Sarma, Non-abelian anyons and topological quantum computation, Rev. Mod. Phys. 80, 1083 (2008).

[8] L. Fu and C. L. Kane, Superconducting Proximity Effect and Majorana Fermions at the Surface of a Topological Insulator, Phys. Rev. Lett. 100, 096407 (2008).

[9] M. Sato, Y. Takahashi, and S. Fujimoto, Non-Abelian Topological Order in $s$-Wave Superfluids of Ultracold Fermionic Atoms, Phys. Rev. Lett. 103, 020401 (2009).

[10] J. D. Sau, R. M. Lutchyn, S. Tewari, and S. Das Sarma, Generic New Platform for Topological Quantum Computation Using Semiconductor Heterostructures, Phys. Rev. Lett. 104, 040502 (2010).

[11] R. M. Lutchyn, J. D. Sau, and S. Das Sarma, Majorana Fermions and a Topological Phase Transition in Semiconductor-Superconductor Heterostructures, Phys. Rev. Lett. 105, 077001 (2010).

[12] Y. Oreg, G. Refael, and F. von Oppen, Helical Liquids and Majorana Bound States in Quantum Wires, Phys. Rev. Lett. 105, 177002 (2010).
[13] J. Alicea, Majorana fermions in a tunable semiconductor device, Phys. Rev. B 81, 125318 (2010).

[14] X.-L. Qi, T. L. Hughes, and S.-C. Zhang, Chiral topological superconductor from the quantum Hall state, Phys. Rev. B 82, 184516 (2010).

[15] S. B. Chung, X.-L. Qi, J. Maciejko, and S.-C. Zhang, Conductance and noise signatures of Majorana backscattering, Phys. Rev. B 83, 100512(R) (2011)

[16] V. Mourik, K. Zuo, S. M. Frolov, S. R. Plissard, E. P. A. M. Bakkers, and L. P. Kouwenhoven, Signatures of Majorana fermions in hybrid superconductor-semiconductor nanowire devices, Science 336, 1003 (2012).

[17] A. Das, Y. Ronen, Y. Most, Y. Oreg, M. Heiblum, and H. Shtrikman, Zero-bias peaks and splitting in an Al-InAs nanowire topological superconductor as a signature of Majorana fermions, Nat. Phys. 8, 887 (2012).

[18] M. T. Deng, C. L. Yu, G. Y. Huang, M. Larsson, P. Caroff, and H. Q. Xu, Anomalous zero-bias conductance peak in a $\mathrm{Nb}-\mathrm{InSb}$ nanowire-Nb hybrid device, Nano Lett. 12, 6414 (2012).

[19] M.-X. Wang, C. Liu, J.-P. Xu, F. Yang, L. Miao, M.-Y. Yao, C. L. Gao, C. Shen, X. Ma, X. Chen, Z.-A. Xu, Y. Liu, S.C. Zhang, D. Qian, J.-F. Jia, and Q.-K. Xue, The coexistence of superconductivity and topological order in the $\mathrm{Bi}_{2} \mathrm{Se}_{3}$ thin films, Science 336, 52 (2012).

[20] S. Nadj-Perge, I. K. Drozdov, J. Li, H. Chen, S. Jeon, J. Seo, A. H. MacDonald, B. A. Bernevig, and A. Yazdani, Observation of Majorana fermions in ferromagnetic atomic chains on a superconductor, Science 346, 602 (2014).

[21] J.-P. Xu, C. Liu, M.-X. Wang, J. Ge, Z.-L. Liu, X. Yang, Y. Chen, Y. Liu, Z.-A. Xu, C.-L. Gao, D. Qian, F.-C. Zhang, and J.-F. Jia, Artificial Topological Superconductor by the Proximity Effect, Phys. Rev. Lett. 112, 217001 (2014). 
[22] J.-P. Xu, M.-X. Wang, Z. L. Liu, J.-F. Ge, X. Yang, C. Liu, Z. A. Xu, D. Guan, C. L. Gao, D. Qian, Y. Liu, Q.-H. Wang, F.-C. Zhang, Q.-K. Xue, and J.-F. Jia, Experimental Detection of a Majorana Mode in the Core of a Magnetic Vortex Inside a Topological Insulator-Superconductor $\mathrm{Bi}_{2} \mathrm{Te}_{3} / \mathrm{NbSe}_{2}$ Heterostructure, Phys. Rev. Lett. 114, 017001 (2015).

[23] J. Wang, Q. Zhou, B. Lian, and S.-C. Zhang, Chiral topological superconductor and half-integer conductance plateau from quantum anomalous Hall plateau transition, Phys. Rev. B 92, 064520 (2015).

[24] H.-H. Sun, K.-W. Zhang, L.-H. Hu, C. Li, G.-Y. Wang, H.-Y. Ma, Z.-A. Xu, C.-L. Gao, D.-D. Guan, Y.-Y. Li, C. Liu, D. Qian, Y. Zhou, L. Fu, S.-C. Li, F.-C. Zhang, and J.-F. Jia, Majorana Zero Mode Detected with Spin Selective Andreev Reflection in the Vortex of a Topological Superconductor, Phys. Rev. Lett. 116, 257003 (2016).

[25] Q. L. He, L. Pan, A. L. Stern, E. C. Burks, X. Che, G. Yin, J. Wang, B. Lian, Q. Zhou, E. S. Choi, K. Murata, X. Kou, Z. Chen, T. Nie, Q. Shao, Y. Fan, S.-C. Zhang, K. Liu, J. $\mathrm{Xia}$, and K. L. Wang, Chiral Majorana fermion modes in a quantum anomalous Hall insulator-superconductor structure, Science 357, 294 (2017).

[26] G. C. Ménard, S. Guissart, C. Brun, R. T. Leriche, M. Trif, F. Debontridder, D. Demaille, D. Roditchev, P. Simon, and T. Cren, Two-dimensional topological superconductivity in $\mathrm{Pb} / \mathrm{Co} / \mathrm{Si}(111)$, Nat. Commun. 8, 2040 (2017).

[27] H. Zhang et al., Quantized Majorana conductance, Nature (London) 556, 74 (2018).

[28] D. Wang, L. Kong, P. Fan, H. Chen, S. Zhu, W. Liu, L. Cao, Y. Sun, S. Du, J. Schneeloch, R. Zhong, G. Gu, L. Fu, H. Ding, and H.-J. Gao, Evidence for Majorana bound states in an ironbased superconductor, Science 362, 333 (2018).

[29] T. Machida, Y. Sun, S. Pyon, S. Takeda, Y. Kohsaka, T. Hanaguri, T. Sasagawa, and T. Tamegai, Zero-energy vortex bound state in the superconducting topological surface state of Fe (Se, Te), Nat. Mater. 18, 811 (2019).

[30] Z. Wang, J. O. Rodriguez, L. Jiao, S. Howard, M. Graham, G. D. Gu, T. L. Hughes, D. K. Morr, and V. Madhavan, Evidence for dispersing 1D Majorana channels in an iron-based superconductor, Science 367, 104 (2020).

[31] L. Fu and E. Berg, Odd-Parity Topological Superconductors: Theory and Application to $\mathrm{Cu}_{x} \mathrm{Bi}_{2} \mathrm{Se}_{3}$, Phys. Rev. Lett. 105, 097001 (2010).

[32] M. Sato, Topological odd-parity superconductors, Phys. Rev. B 81, 220504(R) (2010).

[33] P. Hosur, P. Ghaemi, R. S. K. Mong, and A. Vishwanath, Majorana Modes at the Ends of Superconductor Vortices in Doped Topological Insulators, Phys. Rev. Lett. 107, 097001 (2011).

[34] L. Fu, Odd-parity topological superconductor with nematic order: Application to $\mathrm{Cu}_{x} \mathrm{Bi}_{2} \mathrm{Se}_{3}$, Phys. Rev. B 90, 100509(R) (2014).

[35] P. Hosur, X. Dai, Z. Fang, and X.-L. Qi, Time-reversalinvariant topological superconductivity in doped Weyl semimetals, Phys. Rev. B 90, 045130 (2014).

[36] S. Kobayashi and M. Sato, Topological Superconductivity in Dirac Semimetals, Phys. Rev. Lett. 115, 187001 (2015).

[37] Z. Wang, P. Zhang, G. Xu, L. K. Zeng, H. Miao, X. Xu, T. Qian, H. Weng, P. Richard, A. V. Fedorov, H. Ding, X. Dai, and Z. Fang, Topological nature of the $\mathrm{FeSe}_{0.5} \mathrm{Te}_{0.5}$ superconductor, Phys. Rev. B 92, 115119 (2015).

[38] G. Xu, B. Lian, P. Tang, X.-L. Qi, and S.-C. Zhang, Topological Superconductivity on the Surface of Fe-Based Superconductors, Phys. Rev. Lett. 117, 047001 (2016).

[39] Y. Pan, A. M. Nikitin, G. K. Araizi, Y. K. Huang, Y. Matsushita, T. Naka, and A. de Visser, Rotational symmetry breaking in the topological superconductor $\mathrm{Sr}_{x} \mathrm{Bi}_{2} \mathrm{Se}_{3}$ probed by upper-critical field experiments, Sci. Rep. 6, 28632 (2016).

[40] K. Matano, M. Kriener, K. Segawa, Y. Ando, and Guo-qing Zheng, Spin-rotation symmetry breaking in the superconducting state of $\mathrm{Cu}_{x} \mathrm{Bi}_{2} \mathrm{Se}_{3}$, Nat. Phys. 12, 852 (2016).

[41] S. Yonezawa, K. Tajiri, S. Nakata, Y. Nagai, Z. Wang, K. Segawa, Y. Ando, and Y. Maeno, Thermodynamic evidence for nematic superconductivity in $\mathrm{Cu}_{x} \mathrm{Bi}_{2} \mathrm{Se}_{3}$, Nat. Phys. 13, 123 (2017).

[42] P. Zhang, K. Yaji, T. Hashimoto, Y. Ota, T. Kondo, K. Okazaki, Z. Wang, J. Wen, G. D. Gu, H. Ding, and S. Shin, Observation of topological superconductivity on the surface of an ironbased superconductor, Science 360, 182 (2018).

[43] T. Yoshida and Y. Yanase, Topological $d+p$-wave superconductivity in Rashba systems, Phys. Rev. B 93, 054504 (2016).

[44] A. Daido and Y. Yanase, Paramagnetically induced gapful topological superconductors, Phys. Rev. B 94, 054519 (2016).

[45] O. Can, T. Tummuru, R. P. Day, I. Elfimov, A. Damascelli, and M. Franz, High-temperature topological superconductivity in twisted double-layer copper oxides, Nat. Phys. 17, 519 (2021).

[46] A. P. Schnyder, S. Ryu, A. Furusaki, and A. W. W. Ludwig, Classification of topological insulators and superconductors in three spatial dimensions, Phys. Rev. B 78, 195125 (2008).

[47] A. Kitaev, Periodic table for topological insulators and superconductors, AIP Conf. Proc. 1134, 22 (2009).

[48] S. Ryu, A. P. Schnyder, A. Furusaki, and A. W. W. Ludwig, Topological insulators and superconductors: Tenfold way and dimensional hierarchy, New J. Phys. 12, 065010 (2010).

[49] L. Fu, Topological Crystalline Insulators, Phys. Rev. Lett. 106, 106802 (2011).

[50] F. Zhang, C. L. Kane, and E. J. Mele, Topological Mirror Superconductivity, Phys. Rev. Lett. 111, 056403 (2013).

[51] C.-K. Chiu, H. Yao, and S. Ryu, Classification of topological insulators and superconductors in the presence of reflection symmetry, Phys. Rev. B 88, 075142 (2013).

[52] T. Morimoto and A. Furusaki, Topological classification with additional symmetries from Clifford algebras, Phys. Rev. B 88, 125129 (2013).

[53] K. Shiozaki and M. Sato, Topology of crystalline insulators and superconductors, Phys. Rev. B 90, 165114 (2014).

[54] C.-K. Chiu and A. P. Schnyder, Classification of reflectionsymmetry-protected topological semimetals and nodal superconductors, Phys. Rev. B 90, 205136 (2014).

[55] Y. Ueno, A. Yamakage, Y. Tanaka, and M. Sato, SymmetryProtected Majorana Fermions in Topological Crystalline Superconductors: Theory and Application to $\mathrm{Sr}_{2} \mathrm{RuO}_{4}$, Phys. Rev. Lett. 111, 087002 (2013).

[56] Y. Tsutsumi, M. Ishikawa, T. Kawakami, T. Mizushima, M. Sato, M. Ichioka, and K. Machida, $\mathrm{UPt}_{3}$ as a topological crystalline superconductor, J. Phys. Soc. Jpn. 82, 113707 (2013). 
[57] T. Yoshida, M. Sigrist, and Y. Yanase, Topological Crystalline Superconductivity in Locally Noncentrosymmetric Multilayer Superconductors, Phys. Rev. Lett. 115, 027001 (2015).

[58] C. Fang and L. Fu, New classes of three-dimensional topological crystalline insulators: nonsymmorphic and magnetic, Phys. Rev. B 91, 161105(R) (2015).

[59] K. Shiozaki, M. Sato, and K. Gomi, $Z_{2}$ topology in nonsymmorphic crystalline insulators: Möbius twist in surface states, Phys. Rev. B 91, 155120 (2015).

[60] K. Shiozaki, M. Sato, and K. Gomi, Topology of nonsymmorphic crystalline insulators and superconductors, Phys. Rev. B 93, 195413 (2016).

[61] H. Shapourian, Y. Wang, and S. Ryu, Topological crystalline superconductivity and second-order topological superconductivity in nodal-loop materials, Phys. Rev. B 97, 094508 (2018).

[62] Y. Yanase and K. Shiozaki, Möbius topological superconductivity in $\mathrm{UPt}_{3}$, Phys. Rev. B 95, 224514 (2017).

[63] A. Daido, T. Yoshida, and Y. Yanase, $\mathbb{Z}_{4}$ Topological Superconductivity in UCoGe, Phys. Rev. Lett. 122, 227001 (2019).

[64] S. Ono, Y. Yanase, and H. Watanabe, Symmetry indicators for topological superconductors, Phys. Rev. Research 1, 013012 (2019).

[65] S. Ono, H. C. Po, and H. Watanabe, Refined symmetry indicators for topological superconductors in all space groups, Sci. Adv. 6, eaaz8367 (2020).

[66] S. Ono, H. C. Po, and K. Shiozaki, $\mathbb{Z}_{2}$-enriched symmetry indicators for topological superconductors in the 1651 magnetic space groups, Phys. Rev. Research 3, 023086(R) (2021).

[67] A. Skurativska, T. Neupert, and M. H. Fischer, Atomic limit and inversion-symmetry indicators for topological superconductors, Phys. Rev. Research 2, 013064 (2020).

[68] M. Geier, P. W. Brouwer, and L. Trifunovic, Symmetry-based indicators for topological Bogoliubov-de Gennes Hamiltonians, Phys. Rev. B 101, 245128 (2020).

[69] K. Shiozaki, Variants of the symmetry-based indicator (2019), arXiv:1907.13632 [cond-mat.mes-hall].

[70] J. Ahn and B.-J. Yang, Higher-order topological superconductivity of spin-polarized fermions, Phys. Rev. Research 2, 012060(R) (2020).

[71] M. Sigrist and K. Ueda, Phenomenological theory of unconventional superconductivity, Rev. Mod. Phys. 63, 239 (1991).

[72] S. Khim, J. F. Landaeta, J. Banda, N. Bannor, M. Brando, P. M. R. Brydon, D. Hafner, R. Küchler, R. Cardoso-Gil, U. Stockert, A. P. Mackenzie, D. F. Agterberg, C. Geibel, and E. Hassinger, Field-induced transition within the superconducting state of $\mathrm{CeRh}_{2} \mathrm{As}_{2}$, Science, 373, 1012 (2021).

[73] R. Joynt and L. Taillefer, The superconducting phases of $\mathrm{UPt}_{3}$, Rev. Mod. Phys. 74, 235 (2002).

[74] D. Braithwaite, M. Vališka, G. Knebel, G. Lapertot, J.-P. Brison, A. Pourret, M. E. Zhitomirsky, J. Flouquet, F. Honda, and D. Aoki, Multiple superconducting phases in a nearly ferromagnetic system, Commun. Phys. 2, 147 (2019).

[75] S. Ran, H. Kim, I.-L. Liu, S. R. Saha, I. Hayes, T. Metz, Y. S. Eo, J. Paglione, and N. P. Butch, Enhancement and reentrance of spin triplet superconductivity in $\mathrm{UTe}_{2}$ under pressure, Phys. Rev. B 101, 140503(R) (2020).

[76] D. Aoki, F. Honda, G. Knebel, D. Braithwaite, A. Nakamura, D. Li, Y. Homma, Y. Shimizu, Y. J. Sato, J.-P. Brison, and J. Flouquet, Multiple superconducting phases and unusual enhancement of the upper critical field in $\mathrm{UTe}_{2}, \mathrm{~J}$. Phys. Soc. Jpn. 89, 053705 (2020).

[77] J. Ishizuka and Y. Yanase, Periodic anderson model for magnetism and superconductivity in $\mathrm{UTe}_{2}$, Phys. Rev. B 103, 094504 (2021).

[78] T. Yoshida, M. Sigrist, and Y. Yanase, Pair-density wave states through spin-orbit coupling in multilayer superconductors, Phys. Rev. B 86, 134514 (2012).

[79] E. G. Schertenleib, M. H. Fischer, and M. Sigrist, Unusual $H-T$ phase diagram of $\mathrm{CeRh}_{2} \mathrm{As}_{2}$ : the role of staggered noncentrosymmetricity, Phys. Rev. Research 3, 023179 (2021).

[80] D. Möckli and A. Ramires, Two scenarios for superconductivity in $\mathrm{CeRh}_{2} \mathrm{As}_{2}$, Phys. Rev. Research 3, 023204 (2021).

[81] A. Ptok, K. J. Kapcia, P. T. Jochym, J. Łażewski, A. M. Oleś, and P. Piekarz, Electronic and dynamical properties of $\mathrm{CeRh}_{2} \mathrm{As}_{2}$ : Role of $\mathrm{Rh}_{2} \mathrm{As}_{2}$ layers and expected orbital order, Phys. Rev. B 104, L041109 (2021).

[82] D. C. Cavanagh, T. Shishidou, M. Weinert, P. M. R. Brydon, and D. F. Agterberg, Non-symmorphic symmetry and fielddriven odd-parity pairing in $\mathrm{CeRh}_{2} \mathrm{As}_{2}$, arXiv:2106.02698 [cond-mat.supr-con].

[83] V. M. Edel'shtein, Characteristics of the Cooper pairing in two-dimensional noncentrosymmetric electron systems, Sov. Phys. JETP (English Translation) 68, 1244 (1989).

[84] V. M. Edelstein, Magnetoelectric Effect in Polar Superconductors, Phys. Rev. Lett. 75, 2004 (1995).

[85] E. Bauer, G. Hilscher, H. Michor, Ch. Paul, E. W. Scheidt, A. Gribanov, Yu. Seropegin, H. Noël, M. Sigrist, and P. Rogl, Heavy Fermion Superconductivity and Magnetic Order in Noncentrosymmetric $\mathrm{CePt}_{3} \mathrm{Si}$, Phys. Rev. Lett. 92, 027003 (2004).

[86] D. F. Agterberg and R. P. Kaur, Magnetic-field-induced helical and stripe phases in Rashba superconductors, Phys. Rev. B 75, 064511 (2007).

[87] E. Bauer and M. Sigrist, Non-centrosymmetric Superconductors: Introduction and Overview, Vol. 847 (Springer Science \& Business Media, New York, 2012).

[88] M. Smidman, M. B. Salamon, H. Q. Yuan, and D. F. Agterberg, Superconductivity and spin-orbit coupling in noncentrosymmetric materials: A review, Rep. Prog. Phys. 80, 036501 (2017).

[89] Y. Saito, Y. Nakamura, M. S. Bahramy, Y. Kohama, J. Ye, Y. Kasahara, Y. Nakagawa, M. Onga, M. Tokunaga, T. Nojima, Y. Yanase, and Y. Iwasa, Superconductivity protected by spin-valley locking in ion-gated $\mathrm{MoS}_{2}$, Nat. Phys. 12, 144 (2016).

[90] R. Wakatsuki and N. Nagaosa, Nonreciprocal current in noncentrosymmetric Rashba superconductors, Phys. Rev. Lett. 121, 026601 (2018).

[91] F. Ando, Y. Miyasaka, T. Li, J. Ishizuka, T. Arakawa, Y. Shiota, T. Moriyama, Y. Yanase, and T. Ono, Observation of superconducting diode effect, Nature (London) 584, 373 (2020).

[92] K. Nogaki and Y. Yanase, Strongly parity-mixed superconductivity in the Rashba-Hubbard model, Phys. Rev. B 102, 165114 (2020).

[93] M. H. Fischer, F. Loder, and M. Sigrist, Superconductivity and local noncentrosymmetricity in crystal lattices, Phys. Rev. B 84, 184533 (2011). 
[94] D. Maruyama, M. Sigrist, and Y. Yanase, Locally noncentrosymmetric superconductivity in multilayer systems, J. Phys. Soc. Jpn. 81, 034702 (2012).

[95] D. Maruyama, M. Sigrist, and Y. Yanase, Spin-orbit coupling in multilayer superconductors with charge imbalance, J. Phys. Soc. Jpn. 82, 043703 (2013).

[96] T. Yoshida, M. Sigrist, and Y. Yanase, Complex-stripe phases induced by staggered Rashba spin-orbit coupling, J. Phys. Soc. Jpn. 82, 074714 (2013).

[97] T. Yoshida, M. Sigrist, and Y. Yanase, Parity-mixed superconductivity in locally non-centrosymmetric system, J. Phys. Soc. Jpn. 83, 013703 (2014).

[98] M. Shimozawa, S. K. Goh, T. Shibauchi, and Y. Matsuda, From Kondo lattices to Kondo superlattices, Rep. Prog. Phys. 79, 074503 (2016).

[99] Y. Nakamura and Y. Yanase, Odd-parity superconductivity in bilayer transition metal dichalcogenides, Phys. Rev. B 96, 054501 (2017).

[100] D. Möckli, Y. Yanase, and M. Sigrist, Orbitally limited pairdensity-wave phase of multilayer superconductors, Phys. Rev. B 97, 144508 (2018).

[101] A. Skurativska, M. Sigrist, and M. H. Fischer, Spin response and topology of a staggered Rashba superconductor, Phys. Rev. Research 3, 033133 (2021).

[102] See Supplemental Material at http://link.aps.org/ supplemental/10.1103/PhysRevResearch.3.L032071 which includes Refs. [72,78,81,106,108], for the explicit analytic expression [see Eq. (S46)].
[103] J. Ishizuka, S. Sumita, A. Daido, and Y. Yanase, InsulatorMetal Transition and Topological Superconductivity in $\mathrm{UTe}_{2}$ from a First-Principles Calculation, Phys. Rev. Lett. 123, 217001 (2019).

[104] K. Momma and F. Izumi, vestA3 for three-dimensional visualization of crystal, volumetric and morphology data, J. Appl. Crystallogr. 44, 1272 (2011).

[105] A. Kokalj, XCRYSDEN-A New program for displaying crystalline structures and electron densities, J. Mol. Graphics Modell. 17, 176 (1999).

[106] P. Blaha, K. Schwarz, G. K. H. Madsen, D. Kvasnicka, J. Luitz, R. Laskowsk, F. Tran, L. Marks, and L. Marks, WIEN 2k: An Augmented Plane Wave + Local Orbitals Program for Calculating Crystal Properties (Techn. Universität Wien, Wien, 2018).

[107] See Supplemental Material at http://link.aps.org/ supplemental/10.1103/PhysRevResearch.3.L032071 for the details of the band calculations, density of states, and orbital weights for each atoms (see Sec. S2).

[108] K. Ishida, (Private communication).

[109] Y. Yanase, Nonsymmorphic Weyl superconductivity in $\mathrm{UPt}_{3}$ based on $E_{2 u}$ representation, Phys. Rev. B 94, 174502 (2016).

[110] See Supplemental Material at http://link.aps.org/ supplemental/10.1103/PhysRevResearch.3.L032071 for the details of our minimal tight-binding model analysis and the demonstration of the emergence of Majorana edge states for all 1D irreducible representations (see Secs. S3 and S4). 